\title{
Characterization and Mapping of Oat Crown Rust Resistance Genes Using Three Assessment Methods
}

\author{
E. W. Jackson, D. E. Obert, M. Menz, G. Hu, J. B. Avant, J. Chong, and J. M. Bonman
}

First, second, fourth, fifth, and seventh authors: U.S. Department of Agriculture-Agricultural Research Service, Small Grains and Potato Germplasm Research Unit, 1691 S. 2700 W., Aberdeen, ID 83210; and third author: Syngenta Seeds SAS 12, chemin de l'Hobit BP27, 31790 Saint-Sauveur, France.

Accepted for publication 30 March 2007.

\begin{abstract}
Jackson, E. W., Obert, D. E., Menz, M., Hu, G., Avant, J. B., Chong, J., and Bonman, J. M. 2007. Characterization and mapping of oat crown rust resistance genes using three assessment methods. Phytopathology 97: 1063-1070.

Resistance is the primary means of control for crown rust of oat (Avena sativa L.), caused by Puccinia coronata f. sp. avenae, and better knowledge of the genetics of resistance will enhance resistance breeding. Disease data were generated in the field and greenhouse for parents and recombinant inbred lines of the Ogle/TAM O-301 (OT) oat mapping population using (i) a new quantitative assay that employs quantitative real-time polymerase chain reaction (q-PCR) to estimate fungal growth in the host, (ii) digital image analysis, and (iii) visual ratings. The objectives of this study were to evaluate each assessment method's ability to map a major gene from cv. Ogle and potential quantitative trait loci (QTL)

contributed by Ogle and TAM O-301. All three assessment methods identified the major gene in Ogle, which was mapped to linkage group OT6. The resolution produced by q-PCR, however, enabled more precise mapping of the major gene. Quantitative analysis indicated that $64 \%$ of the phenotypic variation was accounted for using q-PCR, whereas 41 and $52 \%$ were accounted for using visual and digital assessments, respectively. Data generated by q-PCR permitted identification of QTL on linkage groups OT32, accounting for $6 \%$ of the phenotypic variation, and OT2, accounting for $4 \%$ of the variation. QTL on both OT32 and OT2 were conferred by TAM O-301, one of which (OT2) was indiscernible using data from the visual and digital assessments. The new method of precisely phenotyping crown rust resistance provided a more accurate and thorough means of dissecting resistance in the OT mapping population. Similar methods could be developed and applied to other important cereal rust diseases.
\end{abstract}

Development of cultivars with disease resistance is a primary goal for the control of diseases, particularly cereal rusts. Qualitative or major gene resistance generally has a distinct, easily observable, and simply inherited phenotype. Such qualitative resistance is mapped by classifying progeny in segregating populations as either resistant or susceptible and considering resistance as a "phenotypic marker." Using linkage analysis, the phenotypic marker then is associated with genotypic markers mapped on the same population. In contrast, quantitative disease resistances generally are less distinct, inheritance may not fit models for simple Mendelian traits (34), and they are mapped as quantitative trait loci (QTL), enabling the identification of specific loci for resistance in spite of the genetic complexity of the trait (31). Accurate and precise disease assessment is especially crucial in studies of quantitative disease resistance because the effect of the resistance can be relatively small and may be influenced by environment or plant conditions (34). Quantitative resistance to biotrophic pathogens can be useful in the practical control of diseases because of greater durability (21), and is valuable in control of oat crown rust $(5,17)$.

Traditionally, visual assessments have been used to rate cereal rust diseases and diagrammatic scales have been developed as tools to standardize results (23). Visual ratings are inexpensive and have been widely adopted. One large disadvantage, however, is that such assessments rely on the expertise of the rater and, thus, are subject to bias (19). Recently, digital image analyses have

Corresponding author: E. W. Jackson; E-mail address: ejackson@uidaho.edu

* The $\boldsymbol{e}$-Xtra logo stands for "electronic extra" and indicates that the online version contains supplemental material not included in the print edition. Figure 1 appears in color online.

doi:10.1094/PHYTO-97-9-1063

(C) 2007 The American Phytopathological Society been used to eliminate inconsistencies produced by rater bias $(6,26)$. One example of this was the digital analysis method used by Diaz-Lago et al. (6) to assess crown rust on oat leaves, which they considered an improvement over visual ratings. Two limitations to visual and digital assessments are that they measure only visible signs or symptoms and they rely on distinct color contrast differences between diseased and healthy tissues.

Quantitative real-time polymerase chain reaction (q-PCR) is an accurate and sensitive tool to identify and quantify fungal pathogens within host tissues (9). The q-PCR technique has been used to develop assays for several diseases, including rice blast (27), sudden death syndrome of soybean (7), late blight of potato and tomato (13), and leaf stripe and net blotch of barley (1). Building on this knowledge, a new quantitative assay for crown rust was developed using specific protocols for inoculation, sampling, DNA extraction, and real-time q-PCR (12). The assay improved resolution between oat genotypes with varying levels of resistance compared with visual ratings and digital image analysis of the same genotypes (12).

The new quantitative assay could enhance efforts to map crown rust resistance by more clearly phenotyping plants containing loci with large effects and by identifying loci with small effects that are not distinguishable with less sensitive methods. The objective of this study was to compare the new assay to visual and digital assessments for the ability to characterize and map qualitative and quantitative crown rust resistance in the Ogle/TAM O-301 (OT) oat mapping population (25) using a single Puccinia coronata isolate.

\section{MATERIALS AND METHODS}

Plant material. The OT mapping population was developed by single-seed descent to produce $\mathrm{F}_{6}$ seed. Individual $\mathrm{F}_{6}$ plants were grown in the greenhouse and bulked seed from each plant were 
harvested to produce $\mathrm{F}_{6: 7}$ seed. $\mathrm{F}_{7}$ and then $\mathrm{F}_{8}$ rows were grown to produce the $\mathrm{F}_{6: 9}$ recombinant inbred lines (RILs) used in this study.

Fungal isolate and inoculum production. $P$. coronata isolate CR185 (race NBFB) (4), originally collected in Iowa in 1987, was used in this study. CR185 is avirulent on Ogle, producing infection types characterized as flecks (infection type ;) or small flecks with small uredinia (infection type ;1), and virulent on TAM O301 , producing medium to large uredinia with and without necrosis or chlorosis (infection types 3 to 4). Initially, spores were collected using a mini cyclone collector from a single pustule on the susceptible 'Provena'. The spores were suspended in light mineral oil (Soltrol 170, Isoparaffin) and sprayed onto a single Provena plant. After the mineral oil evaporated, the plant was placed in a dew chamber at $21^{\circ} \mathrm{C}$ in the dark for $21 \mathrm{~h}(24,29)$. The plant then was moved to a growth chamber programmed for $13 \mathrm{~h}$ of light at $25^{\circ} \mathrm{C}$ and $11 \mathrm{~h}$ of darkness at $18^{\circ} \mathrm{C}$. After sporulation (12 to 14 days postinoculation), urediniospores were collected using a vacuum collector, desiccated, and stored at $-80^{\circ} \mathrm{C}$.
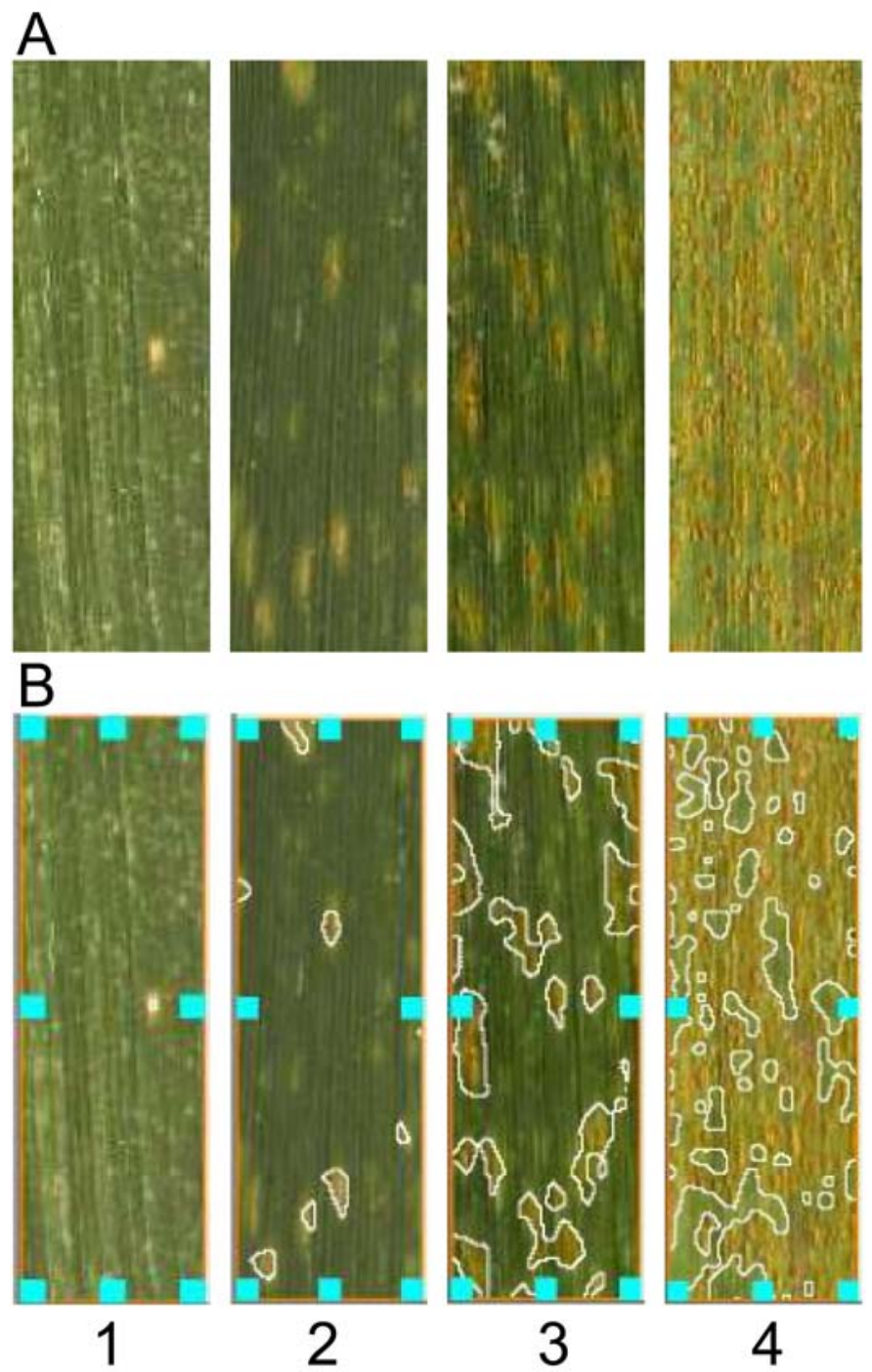

Fig. 1. Phenotypic reactions to Puccinia coronata isolate CR185. A, Examples of scanned images used to estimate percent diseased leaf area (DLA) visually. From left to right, a recombinant inbred line more resistant than Ogle, resistant parent Ogle, susceptible parent TAM O-301, and a recombinant inbred line more susceptible than TAM O-301. B, The same images were used for digital image analysis with Assess image analysis software. Diseased area measured by Assess is indicated by highlighted areas. DLA calculated by the Assess program for each image was $0.12,4.15,32.81$, and $81.37 \%$ for columns 1 through 4 , respectively.
To prepare fresh inoculum for each test, stored urediniospores were suspended in light mineral oil, incubated in a $45^{\circ} \mathrm{C}$ water bath for $10 \mathrm{~min}$, and sprayed onto several Provena plants. The plants were incubated in dew and growth chambers as previously described and, 12 to 14 days, later fresh spores were collected for use in greenhouse and field experiments. For greenhouse evaluations, the spores were suspended in a solution of $0.3 \%$ gelatin and $0.3 \%$ Tween 20 and the final spore concentration was adjusted to $\approx 10^{5}$ urediniospores $\mathrm{ml}^{-1}$. For field evaluations, spores were suspended in light mineral oil and adjusted to $10^{5}$ urediniospores $\mathrm{ml}^{-1}$. All greenhouse and field inoculations were carried out under APHIS permits 64356 and 58342.

Greenhouse experiment. Seed of 88 randomly selected RILs from the original OT population of 136 were sown in $15-\mathrm{cm}$ pots containing a 1:2:1 ( $\mathrm{vol} / \mathrm{vol} / \mathrm{vol})$ mix of sand, peat moss, and vermiculite at 8 seeds per pot. One pot (without replicate) per RIL and two pots per parent were planted and placed in a greenhouse with a 14 - to 15 -h photoperiod at 13 to $21^{\circ} \mathrm{C}$. After emergence, seedlings were thinned to four seedlings per pot. Once plants were at the sixth-leaf stage, a $5-\mathrm{cm}^{2}$ section at the midpoint of each fifth leaf was inoculated with a urediniospore suspension by direct application with sterile nylon swabs as described by Jackson et al. (12). Leaves were allowed to dry and then incubated in the dew chamber and growth chamber as previously described.

Fourteen days after inoculation, sections were excised and scanned at 118 samples/cm. Diseased leaf area (DLA) of each section $(n=368)$ was estimated from digital images using the modified Cobb scale (23) as a reference. Subsequently, DLA also was measured using the Assess image analysis software (15). Using the criteria by Jackson et al. (12) for digital image analysis settings, pustules and a portion of the adjacent chlorotic area were included in the DLA measurement by using threshold settings of 103 and 60 (Fig. 1). The sections with ruptured and unruptured uredinia then were freeze dried and DNA was extracted for estimation of fungal DNA (FDNA) using previously described q-PCR protocols (12).

To allow valid comparisons between the five separate real-time q-PCR runs used to extrapolate FDNA from the 368 greenhouse samples, two replicates of the same FDNA standards were used in each run $(12)$, and replicate samples $(n=4)$ of each genotype were randomized between runs. Comparisons of the slope, intercept, and $R^{2}$ values from regression lines based on cycle threshold (CT) values from the replicate FDNA standards across all plates were made. No data calibration was made before analysis, because standard deviations around the mean regression line equation $\left(y=-3.5 x+34.9, R^{2}=0.997\right)$ were low (slope $=0.1$, intercept $=0.4$, and $R^{2}=0.003$ ).

Field experiment. A randomized complete block design with two replicates was used to evaluate all 136 lines in the OT recombinant inbred (RI) population in the field at the University of Idaho Research and Extension Center in Aberdeen, ID. In each block, 25 to 30 seeds of each line were sown in hill plots. The plots were planted $35.5 \mathrm{~cm}$ apart in rows with 26 plots. Rows were spaced $36 \mathrm{~cm}$ apart with a continuous row of Provena planted every third row as a spreader. Hill plots of each parent were randomly spaced within each row and border plots were planted around each block.

To promote disease development, each block was surrounded by a $6-\mathrm{mm}$ polyethylene sheet attached to a frame. Beginning at the fifth-leaf stage, spreader rows were inoculated at dusk once each week for five consecutive weeks using a hand-held garden sprayer $1 \mathrm{~h}$ after the plots were irrigated. The entire field block then was covered overnight $(12 \mathrm{~h})$ with polyethylene sheeting. After the last inoculation, the blocks were covered four additional times at 3-day intervals to promote disease development and spread from inoculated spreader rows to plots.

Forty-two days after the final inoculation, two flag leaves per plot were selected which best represented the overall disease level 
of each plot (four leaves per RIL, eight leaves per parent). A $5-\mathrm{cm}^{2}$ section at the midpoint of each selected flag leaf was excised and sections were scanned as described for the greenhouse evaluation. Detailed visual estimations of DLA were made from all 560 scanned images (Fig. 1), DLA was calculated using the Assess image analysis software (Fig. 1) and the samples were processed for q-PCR analysis as previously described. Seven separate real-time PCR runs were used to estimate FDNA from the 560 field samples. To allow valid comparison of data from different real-time PCR runs, replicates of parents and RILs were randomized between runs and comparisons were made between the slope, intercept, and $R^{2}$ values calculated from the CT values of replicate FDNA standards used in all runs. As with the greenhouse experiment, no data calibration was made before analysis because the standard deviations around the mean line equation $\left(y=-3.3 x+32.7, R^{2}=0.991\right)$ were low $($ slope $=0.1$, intercept $=$ 0.2 , and $R^{2}=0.003$ ).

Qualitative characterization and mapping. In both experiments, the error from genotype $\times$ "subsample" or replicate (analysis of variance, $P=0.05$ ) (JMP; SAS Institute, Cary, NC) was used to test for significant differences between Ogle and TAM O-301 in DLA and FDNA. Arc-sine transformation of percentage data from DLA assessments was compared with untransformed data. Because transformation did not affect statistical analysis, untransformed data were used. To analyze data from qPCR assessments, $\log _{10}$ transformation was done. The transformation was considered an improvement over untransformed data and was used in analyses.

For each assessment method in each experiment (i.e., six data sets), resistance to CR185 identified in Ogle was qualitatively characterized by classifying RILs as either resistant or susceptible. Because no overlap occurred between the parental disease ranges (Table 1), RILs were classified as resistant if their mean was equal to or below the highest respective DLA or FDNA value measured for the resistant Ogle and as susceptible if greater than the Ogle value. In addition, infection types were used to confirm resistant or susceptible classification of RILs for visual and digital assessments. Based on these criteria, in the greenhouse experiment, RILs with means $\leq 15.0 \%$ DLA (visual), $3.0 \%$ DLA (digital), and $94.5 \mathrm{pg} / \mathrm{mg}$ of oat tissue (q-PCR) were classified as resistant and,

TABLE 1 . Severity of crown rust infection of Ogle and TAM O-301 measured using three different methods after inoculation with Puccinia coronata isolate CR185 in field and greenhouse experiments

\begin{tabular}{llrc}
\hline & & \multicolumn{2}{c}{ Crown rust infection $^{\mathrm{z}}$} \\
\cline { 3 - 4 } Experiment & \multicolumn{1}{c}{ Parent } & Mean & Range \\
\hline Greenhouse & & & \\
Visual & Ogle & $13.8 \mathrm{a}$ & $10.0-15.0$ \\
& TAM O-301 & $57.5 \mathrm{~b}$ & $45.0-80.0$ \\
Digital & Ogle & $1.6 \mathrm{a}$ & $0.9-3.0$ \\
& TAM O-301 & $19.8 \mathrm{~b}$ & $17.0-22.3$ \\
q-PCR & Ogle & $41.1 \mathrm{a}$ & $20.0-94.5$ \\
& TAM O-301 & $1,861.3 \mathrm{~b}$ & $1,028.0-2,230.4$ \\
Field & & & \\
Visual & Ogle & $6.4 \mathrm{a}$ & $5.0-8.0$ \\
& TAM O-301 & $30.4 \mathrm{~b}$ & $12.0-65.0$ \\
Digital & Ogle & $4.5 \mathrm{a}$ & $1.7-8.1$ \\
& TAM O-301 & $46.2 \mathrm{~b}$ & $32.8-73.5$ \\
q-PCR & Ogle & $66.5 \mathrm{a}$ & $26.7-99.8$ \\
& TAM O-301 & $1,299.7 \mathrm{~b}$ & $1,010.9-1,801.9$ \\
\hline
\end{tabular}

y Experiment and assessment.

${ }^{z}$ Mean infection was based on visual estimation and digital image analysis, using the Assess image analysis software of diseased leaf area and quantitative estimation of fungal infection in greenhouse and field evaluations ( $n=8$ plants). Units of measurement were percent diseased area estimated by visual ratings or digital image analysis, and picograms of fungal DNA per milligrams of oat tissue estimated by quantitative real-time polymerase chain reaction (q-PCR). Means within experiment and assessment followed by the same letter are not significantly different $(P=0.05)$. in the field experiment, RILs with means $\leq 8.0 \%$ DLA (visual), $8.1 \%$ DLA (digital), and $99.8 \mathrm{pg} / \mathrm{mg}$ of oat tissue (q-PCR) were classified as resistant. The resulting segregation ratios of resistant/ susceptible RILs for each assessment method per experiment were compared with expected Mendelian ratios and the goodnessof-fit for each comparison was calculated by $\chi^{2}$ tests.

Based on the aforementioned ratios identifying the major gene locus in Ogle, six "low-disease" phenotypic markers were designated (three assessments by two experiments). Using Mapmaker/ Exp 3.0 (16), the phenotypic markers were tested for linkage to molecular markers based on genotypic data from the $\mathrm{F}_{6: 7}$ RIL population used to develop the OT linkage map (25). Linked markers were identified using two-point analysis with a log-likelihood of odds (LOD) score of 4.0 and a maximum distance of 40 centimorgans $(\mathrm{cM})$. A linkage map with the best overall marker order (genotypic and phenotypic) was determined using multipoint linkage analysis with a LOD of 3.0 and a maximum distance of $30 \mathrm{cM}$.

To evaluate the precision of each assessment method in mapping the major gene locus, four different linkage maps (replicates) were calculated for each method per experiment for a total of 24 maps. Mean map distances from restriction fragment length polymorphism (RFLP) probe RZ543 were calculated per method per experiment. Additionally, standard deviations (SDs) around the mean of each map location were calculated.

Quantitative mapping. Resistance in the OT RI population was quantitatively mapped by analyzing data derived from each assessment method per experiment using WinQTL Cartographer (32). QTL analysis was done using a modified OT linkage map with minor marker deletions, insertions, and rearrangements from the original OT map (25). Qualitative evaluation of the data using all assessments identified a major gene reducing crown rust on linkage group (LG) OT6; therefore, composite interval mapping (CIM) was performed to detect both the major loci and additional "nontargeted QTL" sensu Wang et al. (32). Forward regression analyses using the standard CIM model were performed with the recommended settings for control markers (5), window size $(10 \mathrm{cM})$, and walk speed $(2 \mathrm{cM})$. A LOD threshold setting of 3.0

TABLE 2. Reactions to crown rust based on visual, digital, and quantitative polymerase chain reaction (q-PCR) assessments of $\mathrm{F}_{6: 9}$ Ogle $\times$ TAM O-301 recombinant inbred lines (RILs) after inoculation with Puccinia coronata isolate CR185 in field and greenhouse experiments

\begin{tabular}{|c|c|c|c|c|}
\hline \multirow[b]{2}{*}{ Experiment $^{\mathrm{y}}$} & \multicolumn{2}{|c|}{ No. of RILs ${ }^{z}$} & \multicolumn{2}{|c|}{$\chi^{2}(1: 1)$ ratio } \\
\hline & $\mathrm{R}$ & $\mathrm{S}$ & Value & Probability \\
\hline \multicolumn{5}{|l|}{ Greenhouse } \\
\hline Visual & 50 & 38 & 1.64 & $0.25-0.10$ \\
\hline Digital & 46 & 42 & 0.18 & $0.90-0.75$ \\
\hline q-PCR & 43 & 45 & 0.05 & $0.90-0.75$ \\
\hline \multicolumn{5}{|l|}{ Field } \\
\hline Visual & 74 & 62 & 1.05 & $0.50-0.25$ \\
\hline Digital & 69 & 67 & 0.03 & $0.90-0.75$ \\
\hline q-PCR & 67 & 69 & 0.03 & $0.90-0.75$ \\
\hline $\mathrm{F}_{6: 9}$ Total $(\mathrm{df}=6)$ & 349 & 322 & 2.98 & \\
\hline $\mathrm{F}_{6: 9}$ Pooled $(\mathrm{df}=1)$ & $\ldots$ & $\ldots$ & 1.09 & $0.50-0.25$ \\
\hline Heterogeneity $(\mathrm{df}=5)$ & $\ldots$ & $\ldots$ & 1.89 & $0.90-0.75$ \\
\hline
\end{tabular}

y Experiment and assessment. The field experiment was a complete randomized block design with two replications ( $n=136$ lines). The greenhouse experiment had eight subsamples per parent and four subsamples per recombinant inbred line ( $n=88$ lines without replicate). Visual assessments were based on visual ratings from scanned images using the modified Cobb scale as a reference. Digital assessments were done using the Assess imaging software (15), and q-PCR was based on real-time PCR amplifications of total DNA with pathogen-specific primers.

${ }^{\mathrm{z}} \mathrm{R}=$ resistant and $\mathrm{S}=$ susceptible. Resistant lines were those having either diseased leaf area (DLA) or fungal DNA (FDNA) content equal to or less than the upper limit of the resistant parent Ogle, whereas susceptible lines were those having DLA or FDNA greater than the upper limit of Ogle. 
at a significance level of 0.05 was used in all analyses. QTL detected based on data from each assessment were considered valid if they were detected in the same position in both experiments or were cross-validated by two or more assessment methods.

\section{RESULTS}

Disease development. Inoculum levels and environmental conditions were favorable for crown rust development in both field and greenhouse experiments. In both experiments, the susceptible TAM O-301 had significantly higher DLA, as assessed by visual ratings and digital image analyses, and FDNA, as estimated by q-PCR, than did the resistant Ogle (Table 1; Fig. 1). Additionally, TAM O-301 had medium to larger uredinia with and without chlorosis or necrosis, whereas Ogle had flecks with and without small uredinia (Fig. 1). Overall, FDNA estimates by qPCR produced greater relative differences between the two parents than did visual ratings or digital image analysis (Table 1).

Qualitative characterization of resistance. Segregation of the lines in both greenhouse and field experiments and for all three assessment methods did not differ from a 1:1 resistant/susceptible Mendelian model for a single gene (Table 2). In addition, data pooled across experiments and assessment methods fit a single gene model. A test for heterogeneity of the data showed that variability between assessments and experiments was low (Table 2). However, in both experiments, some lines were classified as re- sistant using one assessment method and susceptible using another method. In greenhouse experiments, seven RILs classified as susceptible using FDNA data were classified as resistant using visual ratings. Of these RILs, four also were classified as susceptible using digital image analysis. All seven of these lines had medium-size uredinia with small chlorotic halos. Therefore, no adjustments were made to the original classification. In the field experiment, the seven RILs also were classified as susceptible based on FDNA data that were classified as resistant using visual ratings. Five of these lines also were classified as susceptible using digital image analysis. As in the greenhouse experiments, all seven had medium-size uredinia with small chlorotic halos; thus, no adjustments were made.

Distributions of the RI lines based on visual and digital assessments did not clearly display a bimodal distribution indicative of a 1:1 ratio for a single gene (Fig. 2). Distributions based on visual ratings and digital image analysis in the greenhouse (Fig. 2A and B) and field (Fig. 2C and D) were positively skewed with skewness statistics of 1.1, 1.4, 1.3, and 1.3, respectively (JMP; SAS Institute). In contrast, the distributions of the RILs based on FDNA values from q-PCR in the greenhouse and field better fit a bimodal distribution with skewness statistics of -0.3 and -0.2 , respectively (JMP; SAS Institute) (Fig. 3).

Qualitative mapping. Two-point linkage analysis with a LOD score of 4.0 revealed eight RFLP probes and one amplified fragment length polymorphism (AFLP) marker linked to the candi-

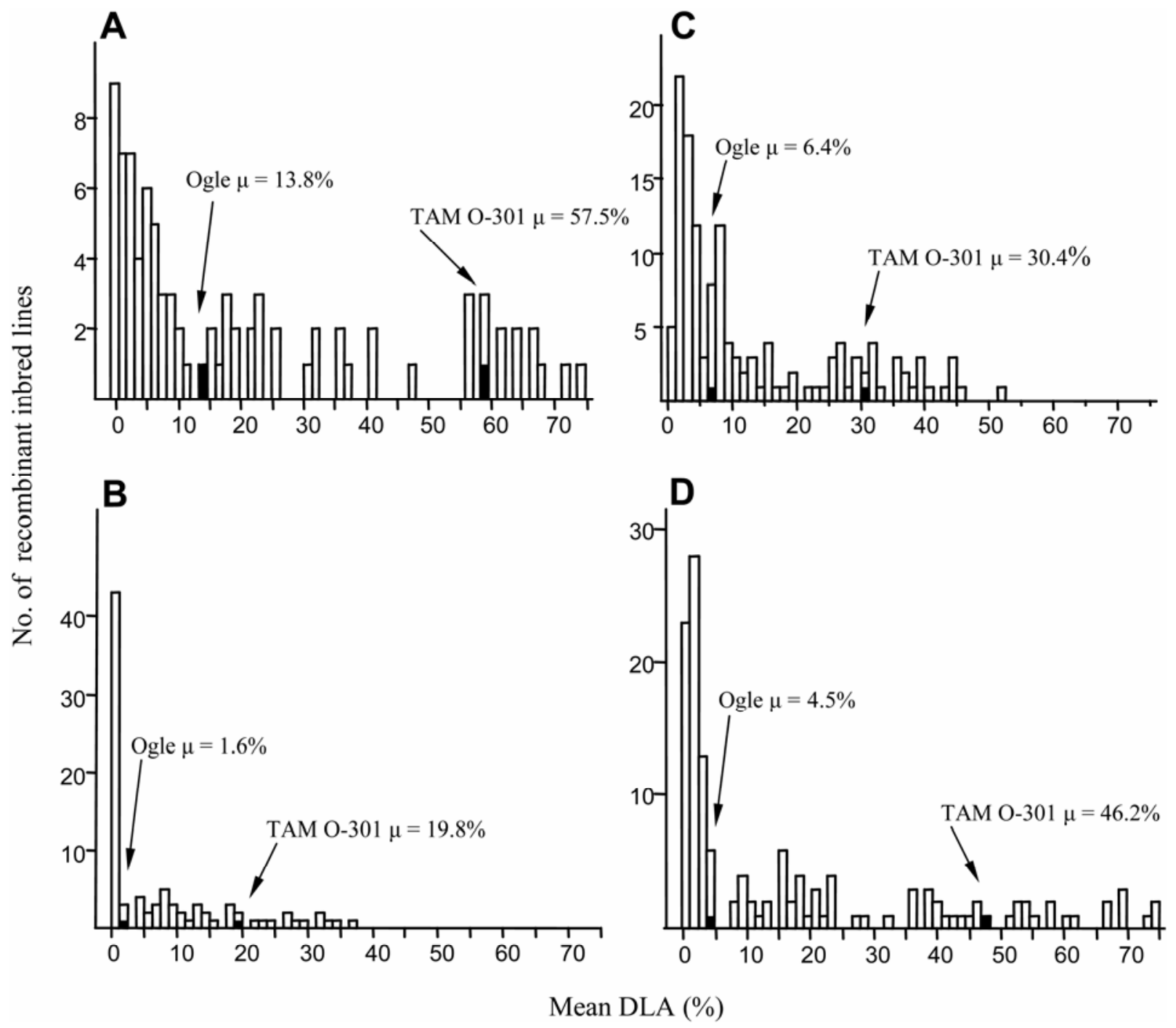

Fig. 2. Frequency distributions of parents and Ogle $\times$ TAM O-301 $\mathrm{F}_{6: 9}$ recombinant inbred lines based on crown rust diseased leaf area (DLA). Distributions are derived from $\mathbf{A}$, visual and $\mathbf{B}$, digital assessments of plants $(n=4)$ inoculated in the greenhouse and $\mathbf{C}$, visual and $\mathbf{D}$, digital assessments in the field. 
date resistance gene. After the nine markers were "grouped," they were broken into subsets and the most likely overall order was determined using the "compare" command with a LOD of 3.0. Using these criteria, 24 different linkage maps were derived for the candidate resistance gene based on phenotypic data from visual, digital, and q-PCR assessments of four individual leaf samples from each experiment. All 24 maps placed the candidate gene in various locations on LG OT6 between RFLP probe RZ543 and AFLP marker e35m61-238.o (Fig. 4). Standard deviations around the mean distances (cM) from RFLP probe RZ543 to the candidate gene locations were calculated for each assessment per experiment (Table 3 ). The mean candidate gene position determined by q-PCR (Fig. 4) had the lowest standard deviation in both experiments (Table 3 ). Because the entire OT population was screened in the field experiment (136 compared with 88), mapping locations were reported using the mean data for each assessment in the field (visual [PcV], digital [PcD], and q-PCR [PcQ] assessments) (Fig. 4).

Quantitative mapping. Composite interval mapping using WinQTL Cartographer (32) detected a major QTL on LG OT6 conferred by Ogle which reduced crown rust severity in both experiments, as expected from qualitative characterization and twopoint linkage analysis (16). The map location, LOD scores, and $R^{2}$ values of this major QTL varied with the disease assessment method used in the analysis $\left(\mathrm{LOD}=11.0, R^{2}=0.41\right.$, visual; 25.0, $R^{2}=0.52$, digital; and 35.0, $R^{2}=0.64$, q-PCR) (Fig. 4). Based on data from visual and q-PCR assessments, the map location of the major QTL overlapped the gene position determined by two-point

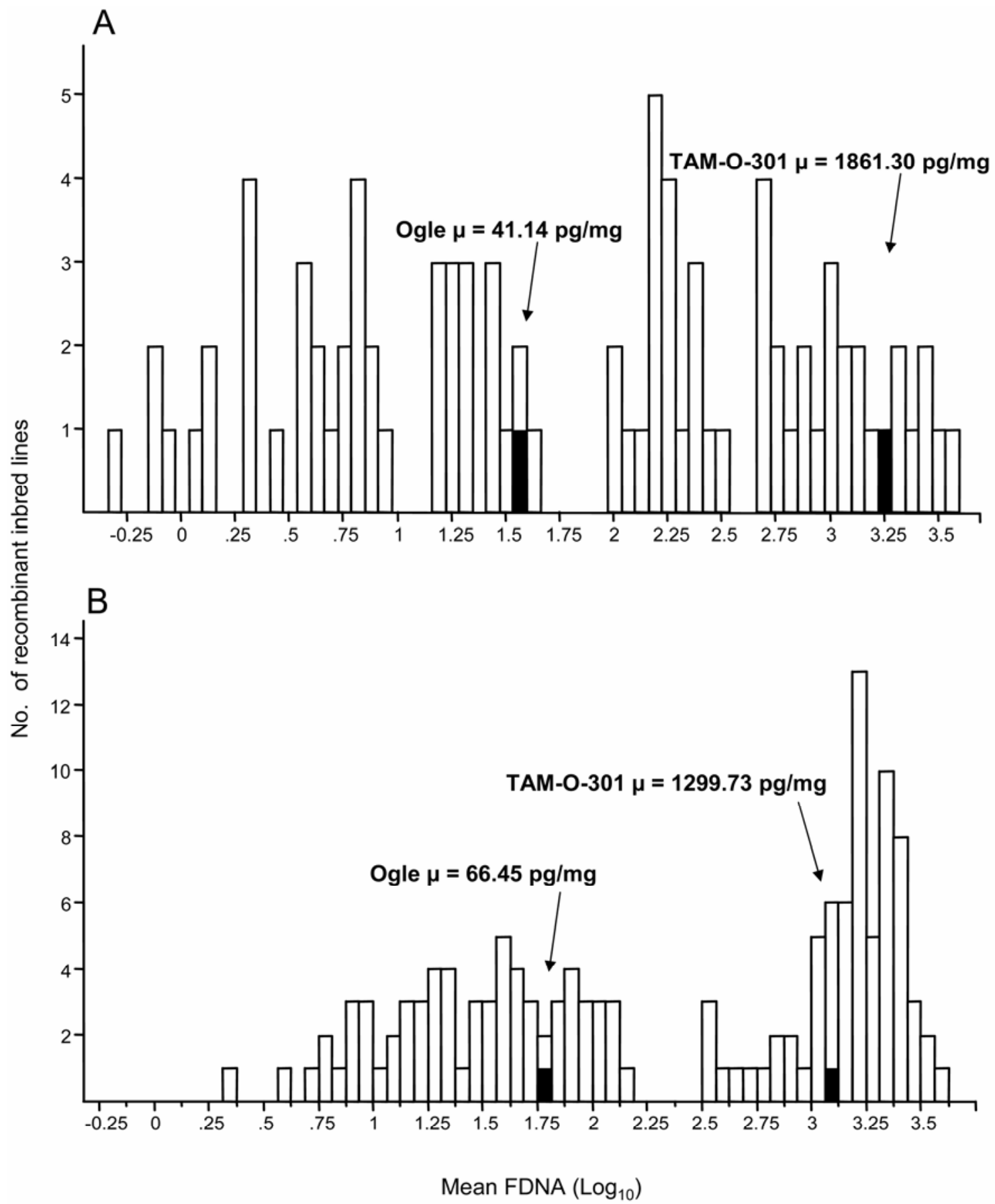

Fig. 3. Frequency distributions of parents and Ogle $\times$ TAM O-301 $\mathrm{F}_{6: 9}$ recombinant inbred lines based on Puccinia coronata fungal DNA (FDNA) in picograms per milligram of oat tissue. Distributions are derived from $\mathbf{A}$, greenhouse and $\mathbf{B}$, field experiments. DNA was extracted from four plants per parent or line in each experiment. 
analyses of the q-PCR data from the field (PcQ) (Fig. 4). In contrast, there was no overlap between the major QTL positions calculated on OT6 and the gene positions assigned by two-point analyses using either visual ratings (PcV) (Fig. 4) or digital image data (PcD) (Fig. 4).

In addition to the major QTL on OT6, CIM detected three other QTL responsible for further reducing crown rust infection conditioned by TAM O-301, one on OT2 and two on OT32, with LOD and $R^{2}$ values of 3.1 and $0.04,4.4$ and 0.06 , and 4.5 and 0.06 , respectively (Fig. 4). The QTL on OT2 was detected only using data from the q-PCR method in both experiments (Fig. 4). Both QTL on OT32 were detected with data from the visual ratings in both experiments and one of these QTL overlapped a QTL detected by the q-PCR assessment of plants grown in the field (Fig. 4).

\section{DISCUSSION}

Using the direct inoculum application method developed by Jackson et al. (12), we were able to generate uniform infection of specific leaf segments using a single $P$. coronata isolate in a greenhouse experiment. Under field conditions, generating uniform infection with single isolates of cereal rust fungi, such as $P$. coronata, can be difficult. In environments that naturally favor disease, other races may contaminate plots whereas, in environments that do not favor disease, such as our testing site, plot conditions must be altered to favor infection by the pathogen. In the present study, adequate disease pressure using a single isolate was produced in the field by inoculating spreader rows in a replicated hill-plot design (22) followed by covering the plots with polyethylene sheeting to provide moisture for infection and subsequent spread of the disease. Providing additional moisture to the plots was crucial for infection at the Aberdeen, ID, location, where the average daily relative humidity during the summer months ranges between 23 and 35\% (National Climatic Data Center, National Oceanic and Atmospheric Administration). During the experiment, no crown-rust-infected plants were found outside the enclosed plot or in surrounding oat plots grown at the University of Idaho Research and Extension Center, indicating that environmental conditions were unfavorable for natural crown
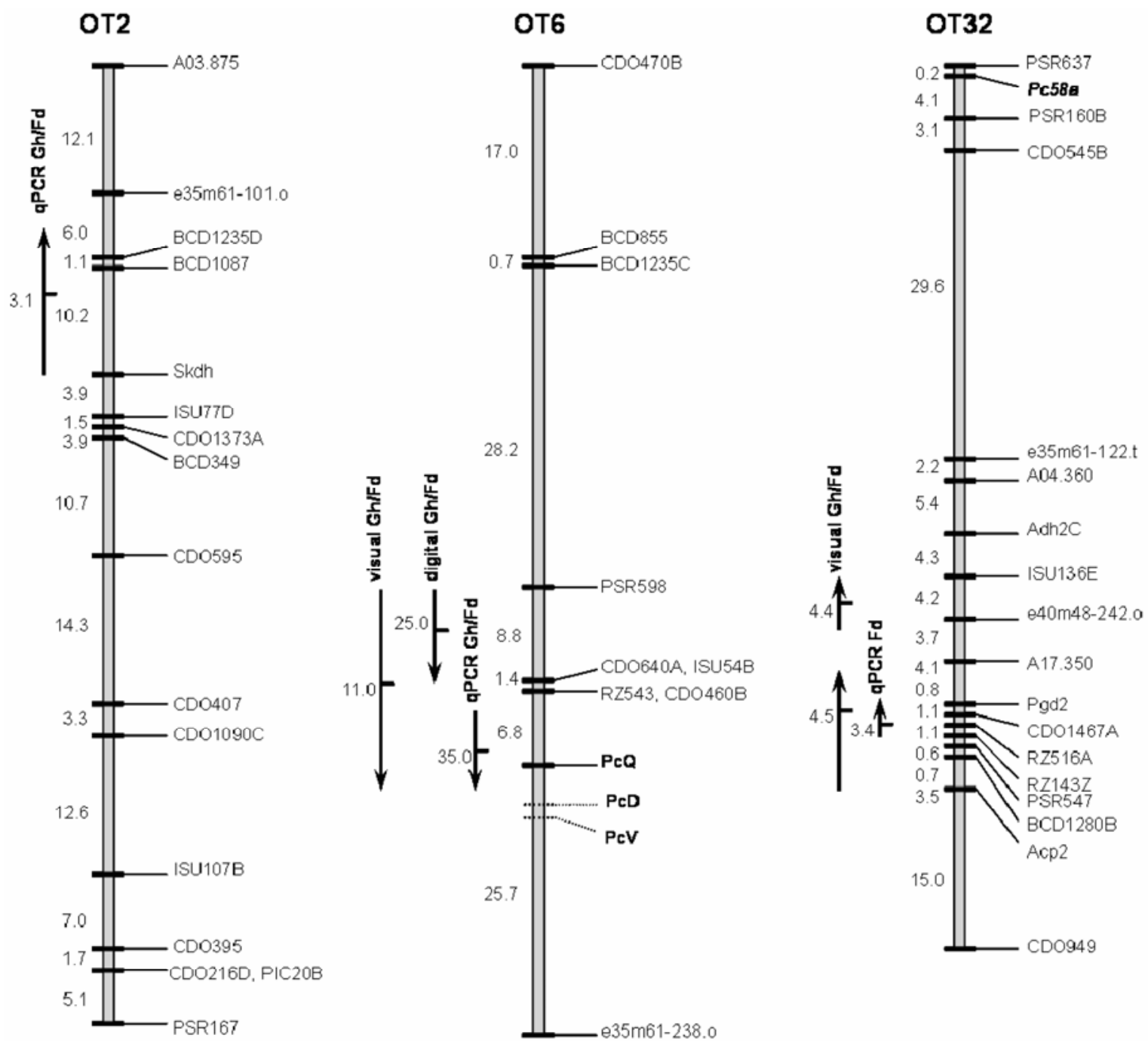

Fig. 4. Ogle $\times$ TAM O-301 (OT) linkage groups showing the candidate resistance gene from Ogle and quantitative trait loci (QTL) regions from TAM O-301 reducing infection by Puccinia coronata. Phenotype is classified based on visual ratings (visual) or digital image analysis (digital) of diseased leaf area (DLA), or fungal DNA (FDNA) content estimated by quantitative real-time polymerase chain reaction (q-PCR) of Ogle $\times$ TAM O-301 $\mathrm{F}_{6: 9}$ recombinant inbred lines inoculated with $P$. coronata isolate CR185 in the greenhouse $(\mathrm{Gh})$ and field $(\mathrm{Fd})$. Genetic markers are based on restriction fragment length polymorphism probes from oat (CDO), barley (BCD), rice (RZ), wheat (PSR), and maize (ISU), amplified fragment length polymorphism markers (a, A), resistance gene analogs (pic 20), and isozyme markers (Idh, Skdh, Acp2, and Pgd2). Genetic distances (left) were generated using Mapmaker 3.0 for each linkage group. The candidate resistance gene positions in Ogle based on visual $(\mathrm{PcV})$, digital $(\mathrm{PcD})$, and $\mathrm{q}-\mathrm{PCR}(\mathrm{PcQ})$ assessments in the field were mapped using two-point linkage analysis with Mapmaker EXP/3.0. Candidate QTL (solid lines to the left of each linkage group) were mapped using WinQTL Cartographer. Arrows indicate whether the allele in Ogle increased $(\uparrow)$ or decreased $(\downarrow)$ DLA or FDNA. 
rust infection in field plots at Aberdeen and that contamination of the experiment from other sources of $P$. coronata was unlikely. By creating a localized epidemic with a single $P$. coronata isolate, it was possible to use the resulting field data along with greenhouse data for genetic analysis of the RI population.

In this study, Ogle was resistant to $P$. coronata isolate CR185 and TAM O-301 was susceptible. TAM O-301 is resistant to most North American crown rust isolates and contains the Pc58 resistance gene cluster $(10,30)$. Ogle is considered susceptible to most $P$. coronata isolates. A previous study, however, showed that Ogle contained two major unlinked resistance loci, which were mapped to the Kanota/Ogle (KO) LGs 4 and 13 (2). Ogle derives $\approx 6.3 \%$ of its genome from 'Landhafer', which contains crown rust resistance genes $P c 4$ and $P c 5$, and $\approx 1.6 \%$ of its genome from 'Victoria', which contains $P c 2, P c 11$, and $P c 12$ (18). Thus, the major resistance gene to CR185 found in the present study could be one of the genes previously identified in Landhafer or Victoria. An allelism test would need to be done to determine if this were true. Additionally, because Ogle is susceptible to most $P$. coronata isolates currently in the United States, the value of this gene to breeding programs may be questionable.

The major resistance gene in Ogle was identified on OT6, which contained six RFLP probes homologous with group $\mathrm{C}$ on the diploid Avena atlanticalA. hirtula map (25). Other studies also have found genetic markers linked to crown-rust-resistant genes which are homologous to group $\mathrm{C}(3,28,35)$. Of these markers found on group C, RFLP probes homologous to LG 13 on the KO map also were linked to crown rust resistance in Ogle $(2,33)$. The $\chi^{2}$ analysis using 70 RILs from KO (2) indicated that resistance in Ogle to $P$. coronata isolate PC54 fit a single-gene model $\left(\chi^{2}=\right.$ $0.1)$. Additionally, analysis on the same RILs inoculated with isolate PC59 fit both single-gene $\left(\chi^{2}=3.4\right)$ and two-gene $\left(\chi^{2}=2.7\right)$ models. The resistant phenotypes conferred by Ogle to both isolates were mapped on $\mathrm{KO} 13$ around $\mathrm{CDO} 460 \mathrm{~A}$ and on $\mathrm{KO} 4$ around UMN214A, with the strongest QTL (LOD 18.0) on KO13. In a separate study, a major gene for resistance to crown rust $(\mathrm{PcC})$ also was mapped on KO13 near CDO460A (L. O'Donoughue, C. Wight, J. Chong, G. Fedak, N. Tinker, and S. Molnar, unpublished data). These previous findings support our data from the present study which linked a major resistance allele from Ogle to CDO460A on OT6. Although only one marker (CDO460A) of the nine on OT6 and KO13 was consistent to both maps, comparative studies with the A. atlantica/A. hirtula map indicate that OT6 and KO13 are on the same chromosome $(20,25,33)$. Therefore, the resistance gene mapped to OT6 in the current study probably is the same as the candidate gene found on KO13 by Bush and Wise (2) and O'Donoughue and associates (L. O'Donoughue, C. Wight, J. Chong, G. Fedak, N. Tinker, and S. Molnar, unpublished data).

Of the assessment methods used to qualitatively map resistance in Ogle, estimates of FDNA using q-PCR were more consistent. This precision probably was due to more accurate classification of RILs. Differences in mean fungal infection between the resistant and susceptible parents measured with q-PCR were 2 to 3 times greater than differences in DLA using digital image analysis and 4 to 10 times greater than differences in DLA measured by visual ratings. The results for digital analysis versus q-PCR were similar to those from a previous study (12). Others also have reported similar improvements in resolution using digital measurements over visual methods $(6,26)$. The effect of greater resolution also was seen in the distribution of RILs from each assessment. Distributions of visual ratings and of DLA values derived from digital image analysis were skewed toward the resistant phenotype, which would not be expected for a simply inherited trait evaluated in the $\mathrm{F}_{6: 9}$ generation. In contrast, distributions from the same samples based on q-PCR, which derived values of FDNA content, were close to bimodal.

A drawback of digital image analysis as applied in this study was the ability to produce timely images with sufficient resolution for detailed analysis. We captured digital images with an HP Scanjet 4070 photosmart scanner at a resolution of 118 samples per $\mathrm{cm}$; however, at this resolution, it was difficult to precisely measure small infected areas with pustules using the Assess image analysis software. Scanning at higher resolution was too slow to be feasible. This difficulty was compounded by less distinct contrast between areas with pustules and those with chlorosis on plants grown in the greenhouse compared with field-grown plants. Thus, the accuracy of digital analysis used in this study perhaps could be improved with better image capture or with the use of more sophisticated image analysis software.

Quantitative mapping based on CIM identified a major QTL conferred by Ogle and additional nontargeted QTL conferred by TAM O-3O1. For the major QTL, the intervals based on data from visual ratings (LOD 11.0) and q-PCR (LOD 35.0) overlapped the position of phenotypic marker PcQ, which was derived from q-PCR assessment of field data and mapped using two-point analysis. In contrast, there was no overlap between the phenotypic markers derived from visual and digital assessments, $\mathrm{PcV}$ and PcD, and the QTL identified via CIM. This result supports the hypothesis that the greater resolution produced by q-PCR improves mapping precision over digital and visual assessments.

In addition to detecting the major gene locus, a QTL on OT2 and a QTL on OT32 were detected using CIM with data based on FDNA content. The QTL on OT2 was not detected with data from either visual ratings or digital image analysis, but was evident in both experiments using the FDNA values. The QTL interval contained the isozyme marker Skdh for shikimate dehydrogenase. Previous studies have linked similar isozymes, including shikimate dehydrogenase, to resistance genes in barley for Barley yellow mosaic virus (14) and in rice for brown planthopper (11). Further studies are being done to confirm and more precisely map this QTL by developing new PCR-based markers in this region.

The second QTL detected on OT 32 using q-PCR data from the field experiment was not detected ( $\geq$ LOD 3.0) in the greenhouse experiment (LOD 2.8). One possible explanation for this was the reduced number of RILs $(n=88)$ tested in the greenhouse. Further greenhouse testing was not deemed necessary because the QTL was validated using data from visual ratings of greenhouse and field experiments. Based on visual ratings, two QTL were detected, one of which directly associated with the QTL identified using q-PCR. It is possible that both QTL identified by visual ratings are part of one distorted QTL interval overlapping the QTL detected by q-PCR. This distortion could be due to reduced resolution of the visual ratings compared with q-PCR.

The QTL interval on OT32 contained the isozyme Acp2 for acid phosphatase, which has been associated with scald resistance in barley (8). Additionally, Portyanko et al. (25) reported that this genetic region was distorted by markers predominantly homo-

TABLE 3. Mean marker positions of a candidate crown rust resistance gene in Ogle based on data from three disease assessment methods of Ogle $\times$ TAM O$301 \mathrm{~F}_{6: 9}$ recombinant inbred lines inoculated with Puccinia coronata isolate CR185 in field and greenhouse experiments ${ }^{\mathrm{y}}$

\begin{tabular}{lcc}
\hline Test $^{\mathrm{z}}$ & Mean marker position (cM) & Standard deviation (cM) \\
\hline Greenhouse & & \\
Visual & 7.3 & 1.3 \\
Digital & 9.6 & 2.8 \\
q-PCR & 9.3 & 0.8 \\
Field & & \\
Visual & 10.9 & 3.1 \\
Digital & 9.9 & 4.7 \\
q-PCR & 6.8 & 0.5 \\
\hline
\end{tabular}

y Marker positions for each test and assessment method are based on a mean centimorgan $(\mathrm{cM})$ distance $(n=4)$ to the rice restriction fragment length polymorphism probe RZ543 on linkage group OT6.

${ }^{\mathrm{z}}$ Experiment and assessment; q-PCR = quantitative real-time polymerase chain reaction. 
logous to TAM O-301. They speculated that the distortion was caused by chromosomal microrearrangements related to the introgression of alien segments carrying disease resistance genes from wild germplasm. Because several RFLP probes exist surrounding the candidate QTL, available genetic information is being used to develop PCR-based markers. If successful, finer mapping of this genetic region will be done to better facilitate the use of this QTL.

Effective control of crown rust by developing resistant cultivars could be achieved by pyramiding several major genes or introgression of quantitative resistance QTL. Marker-assisted selection could make such work feasible. To facilitate this type of selection, useful genetic markers cosegregating with the phenotype must be identified. The present study shows that the accuracy of phenotypic data can be improved by using estimation of FDNA in the host to measure disease levels and, to our knowledge, this is the first study where such measurements have been used to qualitatively and quantitatively characterize and map genes for plant disease resistance. In summary, this work has demonstrated the precision and greater resolution of q-PCR over visual and digital assessments by (i) more precisely characterizing and mapping a major gene using two-point linkage analysis, (ii) consistently and precisely mapping a major gene using Mapmaker EXP/3.0 and QTL Cartographer, (iii) more precisely detecting QTL on OT32 compared with visual ratings, and (iv) identifying a QTL on OT2 indiscernible with other assessment methods.

\section{ACKNOWLEDGMENTS}

We thank K. Satterfield, I. Shackelford, and R. Caldera for their technical expertise in laboratory and field experiments; and D. Wedel, R. Gamble, and the staff of the University of Idaho Research and Extension Center for plot maintenance. This work is dedicated to the memory of David Lee Hoffman, who developed the OT mapping population.

\section{LITERATURE CITED}

1. Bates, J. A., Taylor, E. J. A., Kenyon, D. M., and Thomas, J. E. 2001. The application of real-time PCR to the identification, detection and quantification of Pyrenophora species in barley seed. Mol. Plant Pathol. 2:49-57.

2. Bush, A. L., and Wise, R. P. 1996. Crown rust resistance loci on linkage groups 4 and 13 in cultivated oat. J. Hered. 87:427-432.

3. Bush, A. L., Wise, R. P., Rayapati, P. J., and Lee, M. 1994. Restriction fragment length polymorphisms linked to genes for resistance to crown rust (Puccinia coronata) in near-isogenic lines of hexaploid oat (Avena sativa). Genome 37:823-831.

4. Chong, J., Leonard, K. J., and Salmeron, J. J. 2000. A North American system of nomenclature for Puccinia coronata f. sp. avenae. Plant Dis. 84:580-585.

5. Diaz-Lago, J. E., Stuthman, D. D., and Abadie, T. E. 2002. Recurrent selection for partial resistance to crown rust in oat. Crop Sci. 42:1475-1482.

6. Diaz-Lago, J. E., Stuthman, D. D., and Leonard, K. J. 2003. Evaluation of components of partial resistance to oat crown rust using digital image analysis. Plant Dis. 87:667-674.

7. Gao, X., Jackson, T. A., Lambert, K. M., Li, S., Hartman, G. L., and Niblack, T. L. 2004. Detection and quantification of Fusarium solani $\mathrm{f}$. sp. glycines in soybean roots with real-time quantitative polymerase chain reaction. Plant Dis. 88:1372-1380.

8. Genger, R. K., Nesbitt, K., Brown, A. H. D., Abbott, D. C, and Burdon, J. J. 2005. A novel barley scald resistance gene: Genetic mapping of the Rrs15 scald resistance gene derived from wild barley, Hordeum vulgare ssp. spontaneum. Plant Breed. 124:137-141.

9. Henson, J. M., and French, R. 1993. The polymerase chain reaction and plant disease diagnosis. Annu. Rev. Phytopathol. 31:81-109.

10. Hoffman, D. L., Chong, J., Jackson, E. W., and Obert, D. E. 2006. Characterization and mapping of a crown rust gene complex (Pc58) in TAM O-301. Crop Sci. 46:2630-2635.
11. Huang, N., Parco, A., Mew, T., Magpantay, G., McCouch, S., Guiderdoni, E., and Xu, J. 1997. RFLP mapping of isozymes, RAPD, and QTLs for grain shape, brown planthopper resistance in a doubled haploid rice population. Mol. Breed. 3:105-113.

12. Jackson, E. J., Avant, J. B., Overturf, K. E., and Bonman, J. M. 2006. A quantitative assay of Puccinia coronata f. sp. avenae DNA in Avena sativa. Plant Dis. 90:629-636.

13. Judelson, H. S., and Tooley, P. W. 2000. Enhanced polymerase chain reaction methods for detecting and quantifying Phytophthora infestans in plants. Phytopathology 90:1112-1119.

14. Konishi, T., Ban, T., Iida, Y., and Yoshimi, R. 1997. Genetic analysis of disease resistance to all strains of BaYMV in a Chinese barley landrace, Mokusekko 3. Theor. Appl. Genet. 94:871-877.

15. Lamari, L. 2002. Assess Image Analysis Software for Plant Disease Quantification. American Phytopathological Society, St. Paul, MN.

16. Lander, E. S., Green, P., Abrahamson, J., Barlow, A., Daly, M. J., Lincoln, S. E., and Newberg, L. 1987. Mapmaker: An interactive computer package for constructing primary genetic linkage maps of experimental and natural populations. Genomics 1:174-181.

17. Leonard, K. J. 2002. Oat lines with effective adult plant resistance to crown rust. Plant Dis. 86:593-598.

18. Murphy, H. C., Stanton, T. R, and Coffman, F. A. 1942. Breeding for disease resistance in oats. Agron. J. 34:72-89.

19. Nutter, F. W., Jr., Gleason, M. L., and Christians, N. C. 1993. Assessing the accuracy, intra-rater repeatability, and inter-rater reliability of disease assessment systems. Phytopathology 83:806-812.

20. O’Donoughue, L. S., Wang, Z., Roder, M., Kneen, B., Leggett, M., Sorrells, M. E., and Tanksley, S. D. 1992. An RFLP-based linkage map of oats based on a cross between two diploid taxa (Avena atlantica $\times$ A. hirtula). Genome 33:765-771.

21. Parlevliet, J. E. 2002. Durability of resistance against fungal, bacterial and viral pathogens; present situation. Euphytica 124:147-156.

22. Patanothai, S., Michel, L. J., and Simons, M. D. 1975. Comparison of different hill-plot designs for evaluating quantitative response to oat crown rust. Crop Sci. 15:803-806.

23. Peterson, R. F., Campbell, A. B., and Hannah, A. E. 1948. A diagrammatic scale for estimating rust intensity on leaves and stems of cereals. Can. J. Res. 26:496-500.

24. Politowski, K., and Browning, A. J. 1975. Effect of temperature, light, and dew duration on relative numbers of infection structures of Puccinia coronata avenae. Phytopathology 65:1400-1404.

25. Portyanko, V. A., Hoffman, D. L., Lee, M., and Holland, J. B. 2001. A linkage map of hexaploid oat based on grass anchor DNA clones and its relationship to other oat maps. Genome 44:249-265.

26. Price, T. V, Gross, R., Ho Wey, J., and Osborne, C. F. 1993. A comparison of visual and digital image-processing in quantifying the severity of coffee leaf rust (Hemilea vastatrix). Aust. J. Exp. Agric. 33:97-101.

27. Qi, M., and Yang, Y. 2002. Quantification of Magnaporthe grisea during infection of rice plants using real-time polymerase chain reaction and northern blot/phosphoimaging analyses. Phytopathology 92:870-876.

28. Rayapati, P. J., Gregory, J. W., Lee, M., and Wise, R. P. 1994. A linkage map of diploid Avena based on RFLP loci and a locus conferring resistance to nine isolates of Puccinia coronata var. 'avenae'. Theor. Appl. Genet. 89:831-837.

29. Simons, M. D. 1954. The relationship of temperature and stage of growth to the crown rust reaction of certain varieties of oats. Phytopathology 44:221-224.

30. Simons, M. D., J. W. Martens, R. I. H., McKenzie, I., Nishiyama, K., Sadanaga, Sebesta, J., and Thomas, H. 1978. Oats: A standard system of nomenclature for genes and chromosomes and catalog of genes governing characters. U.S. Dep. Agric. Handb. No. 509.

31. Tanksley, S. D. 1993. Mapping polygenes. Annu. Rev. Genet. 27:205-233.

32. Wang, S., Basten, C. J., and Zeng, Z. B. 2005. Windows QTL Cartographer 2.5. NCSU, Chapel Hill, NC.

33. Wight, C. R., Tinker, N. A., Kianian, S. F., Sorrells, M. E., O'Donoughue, L. S., Hoffman, D. L., and Groh, S. 2003. A molecular marker map in 'Kanota' $\times$ 'Ogle' hexaploid oat (Avena spp.) enhanced by additional markers and a robust framework. Genome 46:28-47.

34. Young, N. D. 1996. QTL mapping and quantitative disease resistance in plants. Annu. Rev. Phytopathol. 34:479-501.

35. Yu, G.-X., Bush, A. L., and Wise, R. P. 1996. Comparative mapping of homoeologous group 1 regions and genes for resistance to obligate biotrophs in Avena, Hordeum, and Zea mays. Genome 39:155-164. 